\title{
Hemorrhagic cardiac tamponade after percutaneous laser ablation of a liver metastasis in segment II
}

\section{Paola Tombesi ${ }^{1}$, Francesca Di Vece ${ }^{1}$, Silvia Rinaldi ${ }^{2}$, Matteo Bertini ${ }^{3}$, Sergio Sartori ${ }^{1}$}

${ }^{1}$ Section of Interventional Ultrasound, Department of Internal Medicine, St. Anna Hospital, 44100 Ferrara, Italy.

${ }^{2}$ Anesthesiology Unit, St. Anna Hospital, 44100 Ferrara, Italy.

${ }^{3}$ Cardiology Unit, St. Anna Hospital, 44100 Ferrara, Italy.

\begin{abstract}
Despite percutaneous laser thermal ablation (LTA) of liver tumors being regarded as a safe technique, major complications can occur. We report the first case of hemorrhagic cardiac tamponade after LTA of a colorectal metastasis in segment II of the liver. Unpredictable heat diffusion causing indirect thermal injury to the pericardium with resultant hemorrhagic reaction was hypothesized as the most likely cause of tamponade. A pericardial drain was emergently placed, $200 \mathrm{~mL}$ of bright red blood were drained, and the patient showed rapid hemodynamic improvement. For lesions located in segment II of the liver and strictly close to the pericardium, a careful risk/benefit analysis should be made by the multidisciplinary team to identify the best treatment option, taking into account both effectiveness and complications of each available technique.
\end{abstract}

Key words: Laser thermal ablation; liver tumors; complications; cardiac tamponade

\section{Corresponding Author:}

Prof. Sergio Sartori, Section of Interventional Ultrasound, Department of Internal Medicine, St. Anna Hospital, Via A. Moro 8, Cona, 44100 Ferrara, Italy. E-mail: srs@unife.it

Received: 29-12-2015, Accepted: 22-04-2016

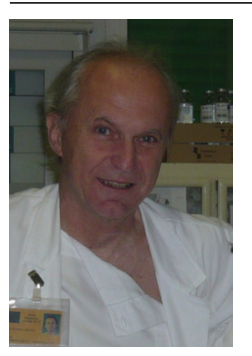

\begin{abstract}
Prof. Sergio Sartori was born on June 22,1954, and graduated cum laude in 1979. He is specialist in Gastroenterology and in Clinical Pharmacology, is dealing with interventional ultrasonography from 1990, and from 2002 is Chief of the Section of Interventional Ultrasound, St. Anna Hospital, Ferrara, Italy. He is author of more than 246 scientific papers.
\end{abstract}

\section{INTRODUCTION}

Percutaneous laser thermal ablation (LTA) of liver malignancies is a well established treatment for both primary and secondary liver tumors, with its effectiveness and safety being proven over the last several years. ${ }^{[1]}$ Among all thermal treatment modalities, LTA enables the use of finer needles than radiofrequency ablation (RFA) and microwave ablation (MWA), and allows one to tailor

\begin{tabular}{|l|c|}
\hline \multicolumn{3}{|c|}{ Access this article online } \\
Website: & Quick Response Code \\
http://www.hrjournal.net/ & \\
DOI: & \\
10.20517/2394-5079.2015.68 & \\
&
\end{tabular}

the ablation volume by using one to four laser fibers, and thus sparing the normal parenchyma as much as possible. These attributes make LTA an attractive option for the treatment of nodules in high-risk locations, and/or multiple nodules differing in size. ${ }^{[2]}$

Although RFA is the most commonly used ablation technique worldwide, the safety profile reported for LTA is comparable to RFA. Mortality rates for both RFA and LTA

This is an open access article distributed under the terms of the Creative Commons Attribution-NonCommercial-ShareAlike 3.0 License, which allows others to remix, tweak, and build upon the worknon-commercially, as long as the author is credited and the new creations are licensed under the identical terms.

For reprints contact: service@oaepublish.com

How to cite this article: Tombesi P, Di Vece F, Rinaldi S, Bertini M, Sartori S. Hemorrhagic cardiac tamponade after percutaneous laser ablation of a liver metastasis in segment II. Hepatoma Res 2016;2:193-6. 


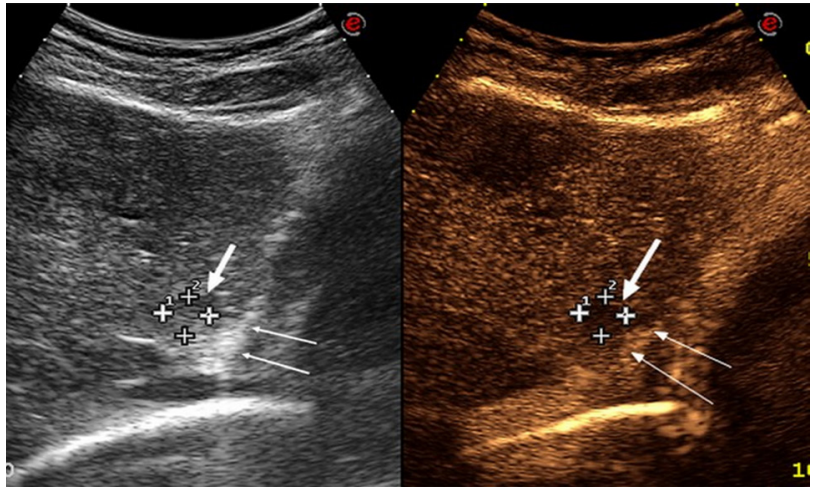

Figure 1: Oblique sub-costal contrast-enhanced ultrasound scan of the left lobe of the liver, showing an $11 \mathrm{~mm}$ metastasis in segment II (large arrows), at close proximity to the diaphragm and pericardium (thin arrows)

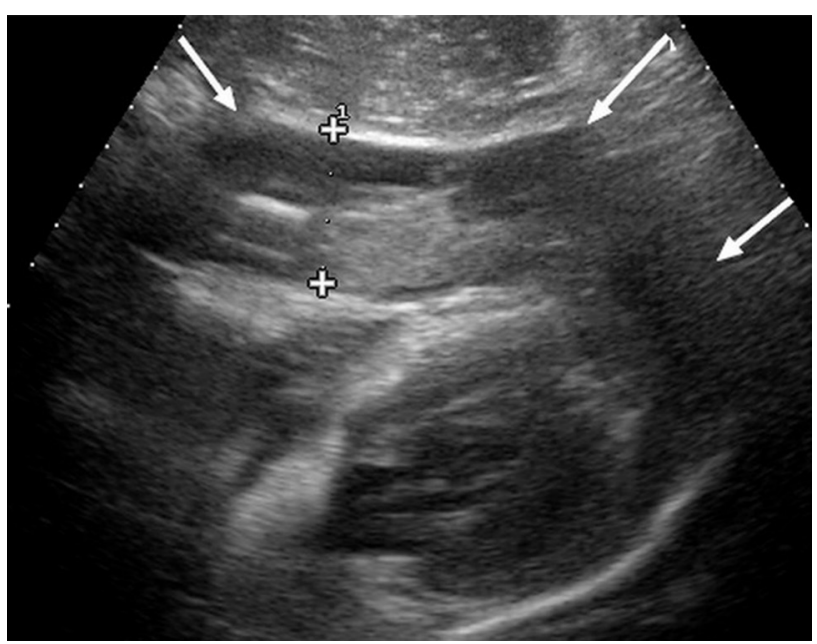

Figure 2: Subxiphoid ultrasound scan showing a large, partially hyperechoic pericardial effusion (arrows) surrounding the cardiac cavities

are less than $1 \%$, and major complication rates range from $3.3 \%$ to $5.1 \%$, and from $1.9 \%$ to $3.5 \%$, respectively. ${ }^{[3-5]}$

Hemorrhagic cardiac tamponade is a very uncommon but potentially fatal complication that has been sporadically reported during RFA of nodules located in the left lobe of the liver, close to the diaphragm and pericardium. ${ }^{[6-8]}$

We report the first case of acute hemorrhagic cardiac tamponade occurring after LTA of a small liver metastasis from colorectal cancer in segment II.

\section{CASE REPORT}

This is a retrospective report of a clinical case, and was exempted from Institutional Review Board approval. The patient gave his written informed consent prior to the interventional procedure.

A 41-year-old man underwent LTA of a small, $11 \mathrm{~mm}$ colorectal metastasis in segment II of the liver, in close proximity to the diaphragm and pericardium [Figure 1]. Four liver metastases in the right lobe and one metastasis in segment III had been successfully ablated by LTA three

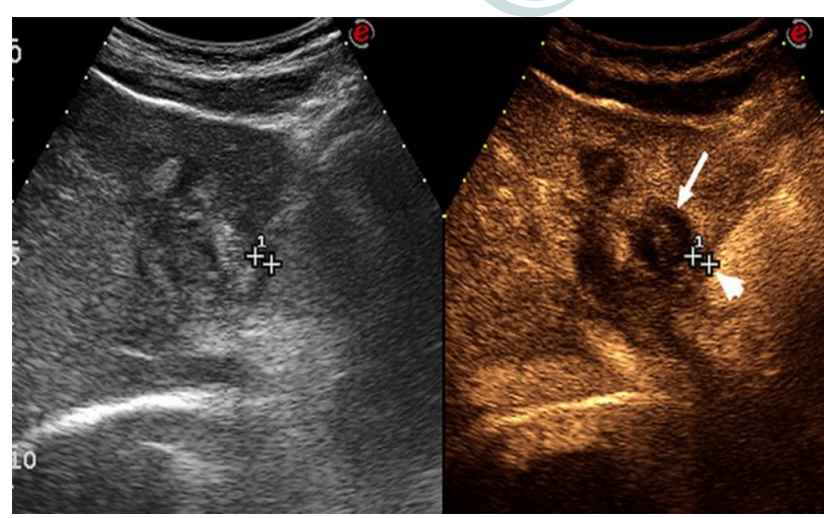

Figure 3: Oblique subcostal contrast-enhanced ultrasound scan of the left lobe of the liver performed a few days after laser thermal ablation, showing complete ablation of the metastasis with a $24 \mathrm{~mm} \times 22 \mathrm{~mm}$ avascular area in segment I (arrows), at a distance of $4 \mathrm{~mm}$ from the diaphragm and pericardium (arrowheads)

months prior, without any complication. The procedure was performed under conscious sedation according to the technique proposed by Pacella et al ${ }^{[9]}$ and modified by Di Costanzo et al. ${ }^{[10]}$ by using a diode laser unit (Echolaser, Elesta srl, Florence, Italy). Under sonographic guidance, two 21-gauge Chiba needles were placed $12 \mathrm{~mm}$ apart from each other along the anterior border of the tumor. Subsequently, two bare-tip $300 \mu \mathrm{m}$ in diameter laser fibers were introduced through the needles and advanced until the tip of the fibers was placed $1 \mathrm{~cm}$ beyond the tip of the needle into the deepest part of the tumor. Eighteen hundred Joule per fiber were delivered in 6 min. Immediately at the conclusion of the procedure, the patient had a sudden episode of tachycardia to 140 beats/min, followed by cardiogenic shock. Ultrasound (US) showed a large amount of partially hyperechoic pericardial fluid [Figure 2]. Cardiopulmonary resuscitation of the patient was initiated, and a 6-French pericardial drain was emergently placed via the paraxiphoid approach by an experienced cardiologist. Two hundred mililiter of bright red blood were drained, and the patient showed rapid hemodynamic improvement. After hemodynamic stabilization, abdominal artery angiography was performed in order to exclude vascular damage to the diaphragmatic arteries and left hepatic artery. No vascular injury was observed, and the patient was admitted to the cardiology unit. He remained asymptomatic, the drainage catheter was removed, and he was discharged after 5 days. Contrast-enhanced US (CEUS) performed before the discharge from the hospital showed complete ablation of the metastasis with a $24 \mathrm{~mm} \times 22 \mathrm{~mm}$ avascular area in segment II [Figure 3]. No lesion or injury of the diaphragm was observed. Echocardiography showed resolution of the pericardial effusion.

Clinical follow up was performed weekly for the first month after discharge, and no further complication was observed.

\section{DISCUSSION}

Acute cardiac tamponade is an extremely infrequent, 
life-threatening complication of thermal ablation treatments. To date, 4 cases of cardiac tamponade have been reported in literature as a complication of percutaneous thermal ablation. ${ }^{[6-8]}$ In all cases, the complication occurred after RFA of liver nodules performed by using expandable radiofrequency needles. The authors hypothesized two possible explanations for the occurrence of cardiac tamponade. ${ }^{[6-8]}$ First, the exact position of expandable RFA needles is more complicated to track at any time than that of the non-expandable RFA probes, MWA antennas, or LTA fibers. Therefore, a RFA hook could have inadvertently been placed in the diaphragm or in the pericardium, causing direct injury to these structures. Indeed, in 2 cases the presence of a RFA hook in the pericardial fat was documented by computed tomography. ${ }^{[8]}$ Secondly, in some unclear circumstances, the distribution of heat in vivo may be unpredictable, and the pericardium can become injured by heat conduction. Indeed, tissues exposed to elevated temperature may react with an inflammatory or hemorrhagic response, and such an injury has been observed in other viscera such as the gall bladder or colon. ${ }^{[11]}$

Although LTA has been less investigated than the other ablation techniques, it seems to have the same efficacy and safety profile as RFA. By using one to four fibers according to the tumor size, the reported complete response rates range from $82 \%$ to $97 \%$ (hazard ratio). Mortality rate is $<1 \%$, and major complication rate ranges from $1 \%$ to $3.5 \%{ }^{[5]}$

To the best of our knowledge, this is the first case of cardiac tamponade following LTA reported in literature. LTA was preferred to other ablation techniques for lesions with small diameters, and those with difficult location. The procedure was performed under US-guidance, which enables one to check the position of the needle in realtime, minimizing the risk of incorrect placement and direct injury to the diaphragm. Moreover, unlike RFA and MWA where the ablation device is advanced through the entire lesion, using LTA technique the advancement of the needle tip was stopped $1 \mathrm{~cm}$ from the deepest part of the tumor, and just the very flexible, flat-tip fibers were placed close to the diaphragm, making direct injury to the diaphragm or pericardium by the needle tip very unlikely. Furthermore, no damage to diaphragmatic arteries or left hepatic arterial vessels was documented by abdominal artery angiography. For all these reasons, even though we cannot exclude with absolute certainty direct damage of pericardium, we believe that the most likely explanation for cardiac tamponade in our patient was unpredictable heat diffusion that caused indirect thermal injury to the pericardium with hemorrhagic reaction. Indeed, CEUS performed a few days after LTA documented successful ablation with a coagulation area $24 \mathrm{~mm} \times 22 \mathrm{~mm}$ in size as expected, indirectly confirming that both needles and laser fibers had been correctly placed into the tumor.
Regardless of the exact mechanism responsible for hemorrhagic cardiac tamponade in our patient, this case report highlights some issues that should be considered in future similar cases. First, in all four cases previously reported in literature as well as in our patient, such a lifethreatening complication occurred with tumors located in segment II of the liver. ${ }^{[6-8]}$ Although cardiac tamponade is an extremely infrequent complication of thermal ablation and is more likely to occur when expandable RFA needles are used ${ }^{[6-8]}$ our experience shows that it may also occur with other theoretically safer techniques, such as LTA. Therefore, tumor location in segment II must be considered a major risk factor for cardiac tamponade during ablation procedures regardless of technique used, and according to Moumouh et al. ${ }^{[6]}$ we wonder: "was percutaneous thermal ablation the best therapeutic option in this case?" A careful risk/benefit analysis must be made ideally by the multidisciplinary team before treating tumors located in segment II. Surgical resection or thermal ablation with open or laparoscopic approach could be considered, as they may be easier for isolating the lesion from adjacent critical structures and potentially provide better control of bleeding. ${ }^{[11]}$ However, these approaches are more invasive and not always simple, and the risk of complications due to an open or laparoscopic approach should be weighed against the risk of cardiac tamponade. In addition, alternative locoregional treatments such as transarterial chemoembolization or stereotactic radiotherapy, or non thermal ablation techniques such as ethanol injection in presence of primary liver tumors should be considered.

Second, early detection of cardiac tamponade is pivotal to minimize its clinical magnitude, and US scans of the pericardial space should be promptly performed when blood pressure suddenly drops during thermal ablation of nodules located in segment II. Likewise, careful consideration should be given to the location where the procedure is performed, in order to ensure rapid availability of emergency personnel and emergency resuscitation equipment to properly manage major complications when they occur.

Finally, the treatment planning of a nodule in segment II should include the presence, or at least the immediate availability, of an interventional radiologist or cardiologist very experienced in the placement of pericardial drains.

\section{Financial support and sponsorship}

Nil.

\section{Conflicts of interest}

There are no conflicts of interest.

\section{REFERENCES}

1. Lahat E, Eshkenazy R, Zendel A, Zakai BB, Maor M, Dreznik $\mathrm{Y}$, Ariche A. Complications after percutaneous ablation of liver 
tumors: a systematic review. Hepatobiliary Surg Nutr 2014;3:31723.

2. Tombesi P, Di Vece F, Sartori S. Laser ablation for hepatic metastases from neuroendocrine tumors. AJR Am J Roentgenol 2015;204:W732.

3. Bertot LC, Sato M, Tateishi R, Yoshida H, Koike K. Mortality and complication rates of percutaneous ablative techniques for the treatment of liver tumors: a systematic review. Eur Radiol 2011;21:2584-96.

4. Arienti V, Pretolani S, Pacella CM, Magnolfi F, Caspani B, Francica G, Megna AS, Regine R, Sponza M, Antico E, Di Lascio FM. Complications of laser ablation for hepatocellular carcinoma: a multicenter study. Radiology 2008;246:947-55.

5. Tombesi P, Di Vece F, Sartori S. Radiofrequency, microwave, and laser ablation of liver tumors: time to move toward a tailored ablation technique? Hepatoma Res 2015;1:52-7.

6. Moumouh A, Hannequin J, Chagneau C, Rayeh F, Jeanny A, Weber-Holtzscherer A, Tasu JP. A tamponade leading to death after radiofrequency ablation of hepatocellular carcinoma. Eur Radiol 2005;15:234-7.
7. Gai J, Sun WB, Tong ZC, Ding XM, Ke S. Successful treatment of acute hemorrhagic cardiac tamponade in a patientwith hepatocellular carcinomaduring percutaneous radiofrequency ablation. Chin Med J (Engl) 2010;123:1470-2.

8. Silverman ER, Lai YH, Osborn IP, Yudkowitz FS. Percutaneous radiofrequency ablation of hepatocellular lesions in segment II of the liver: a risk factor for cardiac tamponade. J Clin Anesth 2013;25:587-90.

9. Pacella CM, Bizzarri G, Magnolfi F, Cecconi P, Caspani B, Anelli V, Bianchini A, Valle D, Pacella S, Manenti G, Rossi Z. Laser thermal ablation in the treatment of small hepatocellular carcinoma: results in 74 patients. Radiology 2001;221:712-20.

10. Di Costanzo GG, D'Adamo G, Tortora R, Zanfardino F, Mattera S, Francica G, Pacella CM. A novel guide system to perform percutaneous laser ablation of liver tumors using the multifiber technique. Acta Radiol 2013;54:876-81

11. Rhim H, Dodd GD 3rd, Chintapalli KN, Wood BJ, Dupuy DE, Hvizda JL, Sewell PE, Goldberg SN. Radiofrequency thermal ablation of abdominal tumors: lessons learned from complications. Radiographics 2004;24:41-52. 